\title{
National Conference on Alternatives to Animal Experiments (NCAAE-2018) in Commemoration of Launching of the Society for Alternatives to Animal Experiments (SAAE) in India
}

doi:10.14573/altex.1902191

\section{Introduction}

In India, Bharathidasan University in Tiruchirappalli has played a significant role in the popularization and practice of alternatives to animal experimentation. Since the establishment of the Mahatma Gandhi-Doerenkamp Center (MGDC) for Alternatives to Use of Animals in Life Science Education at Bharathidasan University in 2009 by the Doerenkamp-Zbinden Foundation (DZF), Switzerland (Akbarsha and Pereira, 2010), the Center has worked towards replacing, reducing, and refining animal use in education, research, and testing. The MGDC organized more than 100 hands-on training workshops for college and university teachers and students on digital and simulation models to replace animal use in teaching and research. More than 20 of these workshops were on animal cell culture techniques and in vitro toxicology, four were on 3D tissue constructs for testing chemicals for skin irritation and corrosion (in collaboration with Episkin, Lyon, France), two were on integrated discrete multiple organ co-culture (in collaboration with In Vitro ADMET Labs, Columbia, MD, USA), one was on systems biology (in collaboration with Hamner Institutes for Health Sciences, RTP, NC, USA), and 5 seminar-cum-workshops were on digital and simulation alternatives for physiology and pharmacology for medical students (in collaboration with PeTA-India).

Other institutions and organizations have also undertaken activities aimed at promoting alternatives and reducing animal use in India: The Government of India Committee for the Purpose of Control and Supervision of Experiments on Animals (CPCSEA), the custodian of the Animal Welfare Act of India, has conducted and sponsored conferences on alternatives, and training programs on animal handling and care (Akbarsha et al., 2011). The concepts and practices of alternatives also were discussed in a special plenary session at the $99^{\text {th }}$ Indian Science Congress, held in Bhubaneshwar (Akbarsha et al., 2012). Further conferences and seminars on alternatives include one jointly organized by the Society of Toxicology, India and SEAC, Unilever, at Hindustan Unilever Ltd., Bangalore (Roy and Akbarsha, 2014); and one sponsored by the Indo-French Centre for the Promotion of Advanced Research (CEFIPRA), conducted at the Indian Institute of Technology, Mumbai (Akbarsha et al., 2016). The Japanese Society for Alternatives to Animal Experiments organized the Asian Congress of Alternatives in Japan during 2016, where it was informally suggested to MGDC to establish a society for alternatives to animal experiments in India. The panel discussion of the "First National Congress on Alternatives to Animal Testing", hosted by the National Facility for Biopharmaceuticals and EnvisBE Solutions, Mumbai, in collaboration with EpiSkin Academy, France, discussed this suggestion and recommended launching such a society (Akbarsha et al., 2018).

After the tenure of the DZF to support MGDC ended, the Center was taken over by Bharathidasan University, and the University Grants Commission (UGC), Government of India, sanctioned a grant to run the Centre under the scheme "Centre with Potential for Excellence in Particular Areas" under the name "National Centre for Alternatives to Animal Experiments (NCAAE)". It was decided to organize a National Conference on Alternatives to Animal Experiments to discuss the perspectives of the current state of practice and existing strategies to promote the development of non-animal technologies in India, on a par with the other countries, to better predict human, animal and environmental responses to a wide range of chemicals and pharmaceuticals. The ultimate goal would be to develop a strategic roadmap to guide the country's future investment and policy changes with regard to animal experiments and offer a platform for sharing ideas with international experts in the field.

Finally, the first National Conference of Alternatives for Animal Experiments was held on November 27, 2018, at Jamia Hamdard (a deemed-to-be university), New Delhi with Vice Chancellor Prof. Seyed E. Hasnain as Patron, and Prof. Sheikh Raisuddin, Department of Medical Elementology and Toxicology, as Organizing Secretary. More than 250 delegates participated. On this occasion, the outline and objectives of the Society for Alternatives to Animal Experiments (SAAE) were introduced. It generated enthusiastic response from the the stakeholders.

\section{Inauguration of the National Conference on Alternatives to Animal Experiments (NCAAE-2018)}

Prof. Sheikh Raisuddin, the Organizing Secretary, welcomed the delegates and introduced the background of the conference. He highlighted the research activities of the Department of Medical Elementology \& Toxicology at Jamia Hamdard in the field of alternatives, especially work on neurotoxicological assessment and mechanistic studies on neurological disorders using Caenorhabditis elegans and Drosophila melanogaster mod- 
els. Dr Renu Swarup, Secretary, Department of Biotechnology (DBT), Government of India, was the Chief Guest at the inauguration. She highlighted the support rendered by DBT towards implementation of newer and advanced technologies in biotechnology research including non-animal methods. In vitro, including stem cell applications, and in silico approaches are among the priority methods contributing to humane experimentation. Research, validation, translation, integration, and scalability are greatly encouraged. Special avenues are open to practicing stem cell technologies towards the development of alternative methods. DBT will promote scientists working towards the development of newer methodologies as alternatives. Prof. Yogendra K. Gupta, a renowned experimental as well as regulatory pharmacologist, retired professor from the All Institute of Medical Sciences (AIIMS), New Delhi, and President-designate of SAAE-India, exhorted that the gap between those who rely on animal experiments and those who want non-animal methods must be bridged. Validation of new methods should aim at practicability, predictability, and reproducibility. "If ever experiments on animals are a must, one must qualify and explain the 'must', but shall not use animals without a pertinent need", he said. He stated that animal experiments can indeed be replaced in several contexts. Prof. Mohammad A. Akbarsha, General Secretary-designate of the SAAE-India, former Director of MGDC and the Doerenkamp Chair for India, explained the background, need of such a conference in India and the current status of the SAAE-India. Prof. Syed E. Hasnain, Vice-Chancellor, Jamia Hamdard, highlighted the importance of alternatives in biomedical research and Prof. Ahmed Kamal, Pro-Vice Chancellor, Jamia Hamdard, and Prof. Maheshwar P. Sharma, Dean, School of Chemical \& Life Sciences, Jamia Hamdard, offered felicitation.

\section{Scientific Lectures}

Dr Troy Seidle, Vice-President Research \& Toxicology, Humane Society International, Toronto, Canada, said in his keynote address that in as much as Tox $21 \mathrm{c}$ envisions "animal-free human risk assessment", the Biomedical Research for the $21^{\text {st }}$ Century Collaboration ${ }^{1}$ recognizes "human biology as the gold standard", in other words, research based on human-specific models. The new approach HSI has is a framework that links the initial molecular events in disease pathways and networks with adverse outcomes, akin to the adverse outcome pathway (AOP) approach under development in toxicology. Such a framework could provide a more predictive and effective rubric for understanding disease pathophysiology across levels of biological organization, and for targeting and evaluating new interventions using the growing toolbox of modern, human-specific microphysiological approaches such as 3D tissue models, organoids, organs-on-achip, and computational systems biology modelling. HSI-India, through the BioMed21 Collaboration, is preparing to launch a funding call to engage Indian health scientists to undertake critical reviews of research progress and barriers in their area of expertise, and convene a national workshop to foster strategic sci- entific dialogue and identify actionable consensus recommendations as a further step toward a comprehensive Indian roadmap for $21^{\text {st }}$ century, human-specific health research and funding.

Dr Hajime Kojima, representing the Japanese Centre for Validation of Alternative Methods (JaCVAM) and the Japanese Society for Alternatives to Animal Experiments (JSAAE), in his plenary lecture briefly reviewed the establishment of the Japanese Society, and explained how JSAAE has been promoting the $3 \mathrm{Rs}$ in Japan through a wide variety of domestic activities and international cooperation. He ensured full support to the SAAE-India.

Dr Christian Pellevoisin, Scientific Director, EPISKIN Academy, Lyon, France, in his plenary lecture spoke about "Reconstructed skin models for in vitro hazard and risk assessment of chemicals and cosmetics". Following the European Cosmetics Regulation (2013), Episkin has observed a strong progressive shift to non-animal methods for safety testing of ingredients and cosmetic products also outside Europe. Mechanistic approaches to replace animals are based on in silico, in chemico, and in vitro assays that can model one or more key events of AOP. Such individual in vitro test systems preferably use cells of human origin with a $3 \mathrm{D}$ organization to best mimic the in vivo situation in humans. Reconstructed human epidermis (RHE) presents several advantages for evaluating some safety endpoints. To date, several alternative methods in toxicology have been developed based on in vitro skin: skin penetration, skin corrosion/irritation, phototoxicity, genotoxicity, and sensitization and two alternative methods based on in vitro skin models have been validated as full replacement methods: OECD TG 431 for in vitro skin corrosion and OECD TG 439 for in vitro skin irritation. Other methods based on human reconstructed epidermis and full thickness models are at different stages of the validation process for different toxicological endpoints. Further, Episkin Academy is engaged in supporting research to find further applications for the RHE.

Prof. Eui-Bae Jeung, from the Laboratory of Veterinary Biochemistry and Molecular Biology, College of Veterinary Medicine, Chungbuk National University, Republic of Korea, also associated with the Korean Society of Alternatives to Animal Experiments, spoke on toxicity testing with embryonic stem cells in his plenary lecture. His group developed a new developmental toxicity test (termed EBT) measuring the area of embryoid bodies $(\mathrm{EBs})$ instead of cardiac differentiation of embryonic stem cells. In the assessment of 21 substances, the EB area logarithmically decreased in a dose-dependent manner. The smaller EB area resulted in a decrease in beating ratio during differentiation of ESCs. The EBT-based prediction model showed $90.5 \%$ accuracy. Next generation sequencing indicated that the reduction in $\mathrm{EB}$ area resulted from cell cycle arrest mediated by HDAC2 and CDKN2A. In order to test the reliability of the proposed EBT method, intra-laboratory reproducibility, inter-laboratory mobility, and inter-laboratory reproducibility tests were performed using identity-coded test substances. Further, a new prediction model was standardized. The results of the intra- and inter-laboratory tests were highly accurate (above $80 \%$ ).

Dr Albert P. Li, President, CEO and Co-founder of In Vitro ADMET Laboratories LLC, Columbia, MD and Malden, MA,

1 https://biomed21.org/ 
USA, in his plenary lecture spoke about unique in vitro hepatic and enteric experimental systems for the evaluation of human xenobiotic metabolism and toxicity. Since results from experiments on laboratory animals do not always accurately reflect human effects, primary human cell-based systems have been developed in his laboratory to aid the assessment of human xenobiotic metabolism and toxicity. For safety evaluation, human organ-specific drug metabolism is especially important, as it is required for metabolic activation of nontoxic parent chemicals to toxic metabolites as well as metabolic detoxification of toxic parent compounds. The in vitro human experimental systems include hepatocytes for hepatic events, enterocytes for intestinal events, as well as the patented Integrated discrete Multiple Organ Co-culture (IdMOCTM) system for the whole human organism.

Dr Dipti M. Kapoor, Science Policy Advisor, PeTA India, New Delhi, India, in her plenary lecture on "Alternatives to the use of animals in regulatory and biomedical research in India" stated that of all the research funding for biomedical research and regulatory policy development from the Indian regulatory and governmental agencies, a large portion goes towards animal experimentation, even though an increasing number of studies show that the results of experiments using animals are often not reproducible or translatable to humans. Pound and Bracken (2014) observed that "if research conducted on animals continues to be unable to reasonably predict what can be expected in humans, the public's continuing endorsement and funding of preclinical animal research seems misplaced." Internationally, an evolving trend has shifted efforts away from animal-based research toward the new and rapidly evolving field of human-relevant, non-animal methods. PeTA India has already discussed with the Union Ministers of Science and Technology, and Environment, Forest and Climate Change, Government of India, the strategies for shifting to non-animal methods. The strategic priorities include i) immediately eliminate animal use in areas in which animals have already been shown to be poor and unreliable predictors for human reactions and have impeded progress; ii) conduct critical scientific reviews of animal use to identify the areas in which their use has failed to advance human health and should therefore be phased out; iii) work with agencies and bodies globally to harmonize and promote international acceptance of non-animal testing methods for regulatory toxicity testing requirements; and iv) divert funds from animal studies towards the development of non-animal methods, including areas in which further development, validation, and implementation of non-animal methods are required.

Dr Barry Hardy, President, OpenTox Association and CEO, Douglas Connect GmbH Technology Park, Basel, Switzerland, spoke on "Collaborative development of predictive toxicology and safety assessment resources - connecting people and data for decision-making". He spoke on OpenTox and its principles and OpenRiskNet as a community effort to develop an open engineering infrastructure supporting risk assessment. He also discussed the challenges of and proposed activities to bring people and diverse resources to the same table to accelerate international collaboration that will support conversation and knowledge activities, leading to strong and rewarding outcomes.
Dr Indira Ghosh, School of Computational \& Integrative Sciences, Jawaharlal Nehru University, New Delhi, India, spoke on "Exploring chemical space in predictive toxicology." A novel algorithm (Maxtox) was established to generate chemical descriptors (Maxtox FP) for chemicals using a maximum common substructure (MCS)-based approach and to develop toxicity prediction models using four endpoint-specific datasets, i.e., mutagenicity, carcinogenicity, TD-50 and EPAFHM (EPA fathead minnow acute toxicity). The models can classify a chemical as toxic or nontoxic on the basis of structural similarity with known toxins. The structural fragments responsible for toxicity were also identified. The effect of different parameters, like training set selection approaches and different machine learning methods, on predictive toxicology performances was discussed. The best model for carcinogenicity prediction, i.e., the MaxtoxRFrep_whole carcinogenicity model, was applied to predict the carcinogenic activity of drugs used for the treatment of diabetes with $84 \%$ accuracy. Out of 45 anti-diabetic drugs from Drugbank, $40 \%$ of investigational and $100 \%$ of withdrawn drugs were predicted to have carcinogenic effects, emphasizing the usefulness of early filtering of designed chemicals in drug development using these approaches.

Dr André Kleensang, Centre for Alternatives to Animal Testing (CAAT), Johns Hopkins University, Baltimore, MD, USA, explained that conventional toxicity testing typically involves studying adverse health outcomes in animals subjected to high doses of toxicants with subsequent extrapolation to expected human responses at lower doses. The low-throughput nature of current in vivo toxicity testing approaches, which are largely the same for industrial chemicals, pesticides and drugs, has led to a backlog of more than 80,000 chemicals to which humans are potentially exposed but for which the possible toxicity remains largely unknown. Some recent developments regarding the paradigm shift in human hazard assessment, away from traditional in vivo animal studies and towards new approach methodologies (NAMs), which include in vitro, ex vivo or omic technologies, and in silico and toxicokinetic modelling, were highlighted. The talk addressed some of the challenging barriers to the integration of NAM data into hazard assessment strategies, in particular for complex endpoints such as repeated dose or reproductive toxicity.

Dr Mohammed Idris, CSIR-Centre for Cellular and Molecular Biology (CCMB), Hyderabad, India, spoke on the alternative model organisms, the zebrafish Danio rerio, marine chordates (Ascidian sp.) and echinoderms (Asterias sp.). These organisms are capable of throwing light on molecular mechanisms of regeneration and degeneration, and are amenable to proteomics, transcriptomics and CRISPR-based gene knock-down approaches. Zebrafish is especially appropriate for understanding the molecular and functional mechanism of neurodegeneration due to triplet repeat expansion like in spinocerebellar ataxia, chronic unpredictable stress as in anxiety-related mood disorders, and chemicals as in Parkinson's disease model.

Dr Aamir Nazir, Senior Scientist, Division of Toxicology and Experimental Medicine, CSIR-Central Drug Research Institute (CDRI), Lucknow, India, shared his experience with Caenorhabditis elegans in neurobiology and aging studies. Transgenic, 
RNAi-based knockdown and mutant strains of $C$. elegans are used to identify novel genetic modulators of age-associated Parkinson's and Alzheimer's disease conditions. He explained the association of novel circular RNA molecules with Parkinson's disease. These molecules are known to act as miRNA sponges, modulating the repression function carried out by miRNAs. Considering the multi-factorial nature of Parkinson's disease, it is hypothesized that a common circRNA/microRNA might trigger multiple manifestations of the ailment. These molecules were studied employing a transgenic $C$. elegans strain expressing human $\alpha$-synuclein with a YFP reporter gene. Using RNAse R-exonuclease treatment of total RNA, followed by amplification using divergent primers, the presence of two highly expressed circRNAs in C. elegans was validated. Their sequence was validated and functional genomics studies were carried out with their synthesizing genes. Employing RNAi, the associated endpoints of $\alpha$-synuclein aggregation were studied in the model followed by whole transcriptome analysis employing NextGen sequencing, which revealed a number of interesting targets.

Prof. Mohammad A. Akbarsha, formerly Doerenkamp Chair at MGDC, Bharathidasan University, Tiruchirappalli, India, spoke on using Hydra, a simple fresh water cnidarian, for risk assessment of chemical pollutants. Whole genome sequencing has revealed conserved sequences and signaling pathways. Data demonstrate the suitability of Hydra for toxicity testing of nanomaterials as well as their bulk counterparts. Acute and chronic studies performed with sub-lethal doses of nanoparticles revealed physiological, developmental and behavioral responses in Hydra. Molecular studies have uncovered the underlying mechanisms of toxicity. Transmission electron microscopic (TEM) analysis revealed sub-cellular alterations and accumulation of nanoparticles within the cells of Hydra. The data substantiate use of Hydra as a model organism for ecotoxicity testing. If validated, this approach will be a remarkable contribution from India.

According to Dr Aditya B. Pant, from the CSIR-Indian Institute of Toxicology Research (IITR), Lucknow, India, animal testing must be the "last resort," when alternative methods are available. A proactive development and implementation of alternative methods must be supported to reduce clinical trial failure rates and the ethical burden of animal use in India. Research into the development of alternative approaches has so far resulted in the incorporation of a panel of new cell and tissue culture systems and in silico models. However, their implementation has been hampered, as regulatory acceptance requires time, prior validation, and robust financial and scientific investments. By channelizing funds away from poorly predictive animal models and towards research into human-focused, non-animal technologies, we can both understand human disease biology better and put animal cruelty behind us.

Dr Brinda Poojary, Research \& Toxicology Department of HSI, Hyderabad, India, emphasized that the time is ripe for India to invest in progressive science, in line with the "Make in India" initiative. In addition to investing in research dedicated to $21^{\text {st }}$ century tools in health research and toxicology testing, focus needs to be directed towards training and educating scien- tists, thus encouraging the adoption of human-centric research and testing. While a few regulators have paved the way for innovation and investment into non-animal methods, the biomedical research fraternity has not yet embraced non-animal technologies. Biomedical research and regulatory testing must cooperate for science to advance in India and to allow India to catch up with other countries. A consolidation of this initiative would be to build an expert committee to validate non-animal methods developed in India.

Dr Vijay Pal Singh, Deputy Director of Food Safety and Standard Authority of India (FSSAI), New Delhi, India, enlightened the delegates about reducing animal use in risk assessment of chemicals/additives in foods. Foods can contain many harmful chemical substances, including pesticides, unhealthy additives or other contaminants. Trace amounts of chemicals may be added during production, transport, or storage of food products. Usually, these chemicals are not harmful unless humans are exposed to them for a long time and at high levels. Risk assessment of food chemicals was developed because of the need to make decisions to protect health in the face of scientific uncertainty. It is pertinent that in the risk assessment of food commodities animal experiments are avoided as far as possible.

Dr Adip Roy, Head - Regulatory Affairs, Amway India Enterprises, Gurgaon, India, speaking about "New frontiers in safety and toxicological assessment", said that risk assessment of ingredients in cosmetics is carried out using a multi-step process to identify a safe level of the ingredient that can form a part of a cosmetic formulation. Assessing safety using non-animal methodologies implies developing novel toxicological risk assessment strategies and a fundamental change in the way safety assessments are carried out. To date, there has been considerable progress in the development, validation and acceptance of non-animal approaches for some toxicological endpoints. Several private and government institutions in India are also actively pursuing research for developing alternate methodologies. With continued long-term research investment, this goal is ultimately achievable.

Dr Syed Ziaur Rahman, Faculty In-charge, Alternatives to Animal Experimentation Lab, Department of Pharmacology, Jawaharlal Nehru Medical College, Aligarh Muslim University, Aligarh, India, shared his experience in practicing non-animal methods in teaching and training of experimental pharmacology. His department has been teaching and demonstrating animal experiments through computer-based simulators/software for the last 13 years. The department established an "Alternatives to Animal Experimentation" lab in 2006. This was the first exclusive lab where experimental work on animals was demonstrated by computer aided education in any medical college in India. The department now has 53 software-based pharmacology experiment teaching modules and some of these simulators are accepted in the revised Medical Council curriculum to help undergraduate students learn the concepts of pharmacology in an interactive and user-friendly manner. Dr Rahman has authored the book " $A$ guide to alternatives to animal experiment in pharmacology".

Dr Subrahmanyam Vangala, Chief Executive Officer \& Cofounder, ReaGene Biosciences Private Limited, Bangalore, India, explained the scope of human-on-chip technology. With the 
advent of new human-on-a-chip technologies, there is great hope for designing better clinical trials with successful clinical translation and outcomes.

Dr Ekta Kapoor, National GLP Compliance Monitoring Authority, Department of Science and Technology (DST), Government of India, New Delhi, India, spoke about the OECD perspective of GLP in toxicology, safety and drug development. She presented an overview of the importance of GLP, Mutual Acceptance of Data (MAD) system, genesis of the National GLP Compliance Monitoring Authority (NGCMA), GLP inspections, and growth of GLP test facilities in India including testing on alternative models.

Oral and poster presentations by students and young researchers generated great interest.

\section{Preconference Workshops}

A workshop on "In vitro Toxicology with emphasis on Integrated discrete Multiple Organ Co-culture (IdMOC)" was hosted by the Department of Zoology University of Kerala, Thiruvananthapuram, on November 22-26, 2018. Supported by University of Kerala, Advanced Centre for Regenerative Medicine and Stem Cell Research in Cutaneous Biology (ACRem-Stem), Society for Translational Cancer Research, and Society for Nutraceuticals and Chronic Diseases; and Co-sponsored by NCAAE, Tiruchirappalli, and SAAE-India, 30 participants were selected to undergo the training. IdMOC is an in vitro, cell culture-based experimental model for the study of cell-cell communication involving more than one organ system. It employs cells from multiple organs that are physically separated but interconnected by a medium that mimics the systemic circulation. The IdMOC technology has numerous applications in drug development and toxicology. It is especially useful in finding the pharmacological effect of a drug and its metabolites on target and off-target organs as well as to evaluate drug-drug interactions. The IdMOC system can be used to assess cell type-specific or organ-specific toxicity. Dr Albert P. Li, from AP Sciences Inc, Columbia, MD, USA, and Dr Mohammad A. Akbarsha, Research Coordinator, National College (autonomous), Tiruchirappalli, were the trainers, and Dr Anil Kumar, from Sri Chitrathirunal Institute of Medical Sciences, Thiruvananthapuram, delivered a lecture connecting stem cells with IdMOC technology. Dr Sreejith Parameswara Panicker, Assistant Professor, Department of Zoology, University of Kerala, Thiruvananthapuram, was the Convener and Organizing Secretary.

A second workshop, "Hands-on Workshop on Alternatives and Skin Irritation Test According to OECD TG-439 using Reconstructed Human Epidermis (RHE)" was held on November 26, 2018 at Jamia Hamdard, New Delhi. It was a collaborative venture of Jamia Hamdard, New Delhi, and EPISKIN Academy, Lyon, France, under the aegis of Society for Alternatives to Animal Experiments-India. The program included a presentation of alternatives and hands-on manipulation of RHE models. The training was based on the OECD TG439 for in vitro skin irritation test for chemicals. A total of 28 trainees attended the programme. Prof. Raisuddin extended the laboratory support, Dr Christian Pellevoisin, Scientific Director, Episkin Acadey, was the key resource person, Dr Poonam Goswami and Dr Mirza Masroor Ali Beg, post-doctoral fellows at the Department of Medical Elementology \& Toxicology of Jamia Hamdard, supported the training.

Overall, this first conference generated an enthusiastic response from stakeholders in India and abroad. It is hoped that the next conference will be organized with more scope for presentations by all stakeholders, especially the young researchers.

\section{Acknowledgements}

The conference was supported financially by Humane Society International (HSI), Doerenkamp-Zbinden Foundation (DZF), PeTA-India, and National Centre for Alternatives to Animal Experiments, BDU, Trichy. MediMation (Animation in Medicine), Episkin Academy, Amway, and J\&K Bank also shared the burden. OpenTox, Switzerland, also collaborated in the endeavor. Jamia Hamdard also poured in its resources for success of the event.

\section{References}

Akbarsha, M. A. and Pereira, S. (2010). Mahatma GandhiDoerenkamp Center for alternatives to use of animals in life science education. J Pharmacol Pharmacother 1, 108-110. doi:10.4103/0976-500X.72353

Akbarsha, M. A., Zeeshan, M. and Pereira, S. (2011). CPCSEA national conference, Chennai, India. ALTEX 28, 153-155. doi:10.14573/altex.2011.2.153

Akbarsha, M., Zeeshan, M. and Pereira, S. (2012). Alternatives discussed at Indian science congress. ALTEX 29, 216-218. doi:10.14573/altex.2012.2.216

Akbarsha, M. A., Majumder, A., Misra, N. et al. (2016). Futuristic approach to alternatives. ALTEX 33, 469-470. doi:10.14573/altex.1607251

Akbarsha, M. A., Mascarenhas, B. and Pellevoisin, C. (2018). First national congress on alternatives to animal testing and post-congress workshops in India. ALTEX 35, 258-260. doi:10.14573/altex.1802231

Pound, P. and Bracken, M. B. (2014). Is animal research sufficiently evidence based to be a cornerstone of biomedical research? BMJ 348, g3387. doi:10.1136/bmj.g3387

Roy, A. and Akbarsha, M. A. (2014). Novel strategie for toxicological risk assessments. ALTEX 31, 91-93. doi:10.14573/ altex. 1311081

Mohammad A. Akbarshal, Sheikh Raisuddin ${ }^{2}$ and Dipti M. Kapoor ${ }^{3}$

${ }^{1}$ National College (Autonomous), Tiruchirappalli, India; ${ }^{2}$ Department of Medical Elementology and Toxicology, Jamia Hamdard - Deemed University, New Delhi, India; ${ }^{3}$ People for Ethical Treatment of Animals (PeTA) India, New Delhi, India 